\title{
Criminologie
}

\section{De grandes espérances}

\section{Jean-Paul Brodeur}

Volume 40, numéro 2, automne 2007

Peines et pénalité au Canada. Autour des travaux de Pierre

Landreville

URI : https://id.erudit.org/iderudit/016858ar

DOI : https://doi.org/10.7202/016858ar

Aller au sommaire du numéro

Éditeur(s)

Les Presses de l'Université de Montréal

ISSN

0316-0041 (imprimé)

1492-1367 (numérique)

Découvrir la revue

Citer cet article

Brodeur, J.-P. (2007). De grandes espérances. Criminologie, 40(2), 161-166.

https://doi.org/10.7202/016858ar

Ce document est protégé par la loi sur le droit d'auteur. L'utilisation des services d'Érudit (y compris la reproduction) est assujettie à sa politique d'utilisation que vous pouvez consulter en ligne.

https://apropos.erudit.org/fr/usagers/politique-dutilisation/
Cet article est diffusé et préservé par Érudit.

Érudit est un consortium interuniversitaire sans but lucratif composé de l’Université de Montréal, l'Université Laval et l'Université du Québec à Montréal. Il a pour mission la promotion et la valorisation de la recherche. https://www.erudit.org/fr/ 


\section{De grandes espérances}

Jean-Paul Brodeur

Directeur, Centre international de criminologie comparée (CICC)

École de criminologie, Université de Montréal

jean-paul.brodeur@umontreal.ca

En 1986, Pierre Landreville s'affairait à la préparation du rapport du Comité d'étude sur les solutions de rechange à l'incarcération (août 1986). J'habitais moi-même Ottawa depuis 1984, où j'occupais mes fonctions de directeur de la recherche pour la Commission canadienne sur la détermination des peines. Cette commission remit son rapport en 1987 et celui-ci n'eut pas d'effets plus retentissants que le comité présidé par Pierre Landreville. Nous étions, à cette époque, plus fervents qu'aujourd'hui et plus engagés dans ce qui fut le combat de notre génération: provoquer la régression, sinon l'abolition de l'incarcération et même le démantèlement du système pénal. Le manque de retombées spectaculaires d'un rapport était considéré comme un accident de parcours tant nous étions convaincus que nous finirions par prévaloir. Nos maîtres nous en avaient fourni l'assurance. Pour Nils Christie, le système pénal se rendait coupable d'un vol de conflit en dépossédant les parties à ce conflit de leur trésor de querelle; il fallait donc retrouver le sens des palabres africaines citées en exemple d'une manière plus humaine de régler les différends. Je me souviens également que dans les cours qu'il donnait dans les années 1975 à l'École de criminologie, Louk Hulsman prévoyait que l'incarcération disparaitrait au cours des quinze prochaines années. Je lui avais posé explicitement cette question de l'horizon temporel et avais obtenu cette réponse.

1. Les génocides du Rwanda et du Darfour, de même que l'inimaginable cruauté des guerres en Sierra Leone et au Liberia, ont mis à mal 
la représentation nordique de l'Africain bon palabreur. Quant à l'abolition de l'incarcération, les choses ont mal tourné. Certains pays comme le Canada et les pays scandinaves sont parvenus à grande peine à maintenir la stabilité de leur taux d'incarcération. Le seul pays qui ait réussi à réduire de façon notable sa pratique de l'emprisonnement est la Finlande, qui a adopté une politique pénale inspirée par le Just Desert préconisé par le sulfureux Von Hirsch (renseignements pris auprès d'un collègue finlandais, les taux finlandais se sont remis à croître en 2007). Partout ailleurs, l'incarcération a sensiblement progressé. Toutes formes d'enfermement considérées, il y a plus de deux millions de personnes incarcérées aux États-Unis; au Royaume-Uni, les taux d'incarcération se sont envolés sous un gouvernement travailliste. Qu'en sera-t-il si les conservateurs reviennent au pouvoir? On pourrait allonger cette sombre liste indéfiniment.

Que s'est-il donc passé? Des tas de choses sans doute. Je me limiterai à n'en mentionner que deux. Il faut d'abord écarter l'explication selon laquelle des pratiques de justice pénale qui étaient concevables dans de petits pays réputés pour leur progressisme, tels que la Norvège et les Pays-Bas, n'étaient pas exportables dans des pays plus populeux comme les États-Unis et la Grande-Bretagne. En fait, la montée la plus spectaculaire des taux d'incarcération s'est produite aux Pays-Bas. Le taux d'incarcération dans ce pays était de 18 personnes pour 100000 habitants en 1973; il est monté à 33/100 000 en 1987 ; il est maintenant de 101/100 000 (87/100 000 si on exclut du compte les mineurs incarcérés). Au final, le taux d'incarcération des Pays-Bas s'est multiplié par cinq depuis 1973. Comment expliquer cela? On ne manquera pas d'invoquer l'action de politiciens conservateurs tels que Pim Fortuyn (considéré dans un concours récent tenu en Hollande comme étant le plus grand des Néerlandais, bien devant Rembrandt et Guillaume le Taciturne). Cette explication est en partie valable, mais elle ne touche pas l'essentiel du phénomène. En effet, la montée très sensible de l'incarcération qui s'est produite depuis 1990 a coïncidé avec une chute de la criminalité parfois très prononcée dans la plupart des pays occidentaux. L'explication que je proposerais à titre d'hypothèse est celle-ci. On assiste à une disjonction de plus en plus profonde entre les faits tels qu'on peut tenter de les mesurer objectivement et la perception imaginaire de la réalité par l'opinion publique. Cette disjonction atteint son écart maximum lorsque l'autorité politique déclare qu'il faut appliquer des politiques pénales répressives pour répondre à la demande de 
l'opinion publique, même si celle-ci est mal fondée et repose sur une perception erronée de la réalité. Quelle que soit sa validité, il suffit donc que l'opinion publique se manifeste pour que les autorités politiques s'estiment pieusement contraintes d'y donner des suites pénales (elles ne sont pas toujours pressées de sacrifier à l'opinion publique). On assiste donc à l'énoncé d'un nouveau principe qui parodie une maxime romaine souvent citée. Selon cette maxime, la loi est dure mais c'est la loi (dura lex, sed lex). Elle doit donc être observée. Dans ses nouveaux oripeaux politiciens, cette maxime est ainsi travestie: même si elle est fausse, l'opinion publique demeure l'opinion du public. Il faut donc la suivre (quand cela est expédient).

2. Il est une seconde forme d'errance dont les «progressistes» (parmi lesquels je me range) furent tous et toutes plus ou moins les victimes. L'affirmation que le crime est une réalité construite est en réalité ambiguë et comporte deux interprétations différentes. Considérons pour la clarté du propos qu'une catégorie est comme une boîte dans laquelle on range divers objets. On peut dire en premier lieu que le contenu de cette boîte varie profondément à travers le temps. En appliquant ce raisonnement à la catégorie du crime, on dira que celle-ci a recouvert des comportements très variables dans l'histoire. Par exemple, la dissection des cadavres menait droit au bûcher il n'y a pas si longtemps. Telle est donc une première interprétation de la proposition selon laquelle le crime est une réalité construite: le crime est construit en ce sens que cette étiquette est appliquée à des comportements hétérogènes parmi lesquels on a peine à trouver un «noyau dur». Il existe une interprétation beaucoup plus radicale de la thèse sur le caractère construit du crime. Selon cette interprétation, c'est la catégorie même du crime qui est appelée à disparaître. Pour revenir à notre analogie, on soutient non seulement que le contenu de la boîte est variable, mais aussi que cette boîte doit elle-même être jetée car elle ne remplit aucune fonction assignable. C'est cette seconde interprétation, la plus radicale, qui fut retenue dans les années 1970-1980. En s'appuyant sur le caractère disparate des comportements qui étaient criminalisés, on en vint à conclure que la catégorie même de crime était vouée à l'obsolescence. La suite des événements devait montrer que cette interprétation radicale ne pouvait s'incarner dans la réalité: les comportements criminalisés changent, mais la catégorie du crime demeure, même si son contenu se transforme. C'est ce que Durkheim avait vu avec profondeur dans les 
pages qu'il consacre au crime dans ses Règles de la métbode sociologique. $\mathrm{Si}$, nous dit Durkheim, la société se transformait en un monastère de saints, la catégorie du crime ne s'évanouirait pas: on criminaliserait tout simplement les peccadilles dont les moines ne manqueraient pas de se rendre coupables. D'avoir été trop célébrée, l'utopie d'une civilianisation sans résidu du droit pénal s'est transformée en son contraire. C'est le droit pénal qui colonise le droit civil et non l'inverse.

3. Que de déconvenues! Eût-il été préférable de s'en préserver en réduisant ses interventions au ronron qui s'élève de la litière du criminologue quand il se love dans les vertus du système pénal à pratiquer la résolution de problèmes? Rien n'est moins sûr. On peut s'accorder sur le fait que le meurtre est généralement considéré comme le plus grave des crimes. Dans cette perspective, une accumulation de meurtres constitue donc une criminalité monstrueuse. Tel est bien le cas du génocide. Le système pénal réussit-il à résoudre le problème que constitue le génocide? Vaste question dont je traiterai en raccourci à l'aide d'un récit que j'ai déjà raconté ailleurs et que j’abrégerai pour les besoins de ce texte. Le génocide perpétré au Rwanda en 1994 a fait quelque 600000 morts. Une femme tutsi (Odette) était mariée à un homme hutu (Théophile). Lorsque le génocide se déclencha, Théophile chercha Odette et ses enfants pour les tuer. Cette dernière et ses enfants réussirent à se cacher. Pour se venger, Théophile assassina la mère d'Odette et plusieurs enfants dont elle avait la garde. Théophile fut par la suite arrêté et détenu avec des milliers d'autres massacreurs dans un camp en Tanzanie, en attendant son procès. Odette tenta d'obtenir un divorce de Théophile, qui avait tué sa mère. Le consentement de ce dernier était nécessaire. Il le refusa et le divorce ne se fit pas. Quand elle cessa d'avoir des nouvelles de Théophile, Odette se remaria. Environ neuf ans passèrent. Au terme de cette période, la justice pénale internationale reconnut qu'il lui était impossible de traduire devant les tribunaux tous ceux qui s'étaient rendus coupables de génocide (des milliers de prévenus). Il fut donc décidé de les libérer s'ils reconnaissaient leurs crimes et s'ils participaient à un stage de rééducation de trois mois. Tout le monde s'empressa de reconnaître ses crimes; tout le monde fut dûment rééduqué; puis, tout le monde rentra à la maison. Lorsqu'il revint dans ses foyers, Théophile voulu emménager à nouveau avec sa femme et ses enfants, qu'il considérait comme ses possessions. Ceux-ci s'y refusèrent pour des raisons évidentes. Théophile fut convaincu par des avocats 
d'entreprendre des poursuites pénales contre sa femme, qui s'était techniquement rendue coupable du crime de bigamie, qui figurait en bonne place dans le Code pénal légué par le colon européen et qui était puni de deux ans d'incarcération. Odette fut donc accusée devant les tribunaux, qui prononcèrent un non-lieu ambigu après une longue et coûteuse procédure où s'affrontèrent les vedettes du Barreau de Kigali. Qu'en conclure sur le plan de la capacité du système pénal à résoudre des problèmes dans ces situations extrêmes? Le système pénal rwandais était parfaitement outillé pour prendre en charge les accusations (loufoques) de bigamie et il s'exécuta avec le décorum et les effets de manches que l'on imagine. Par contre, la justice pénale tant rwandaise qu'internationale échoua complètement à rendre quelque justice que ce fût aux victimes du génocide. Cette conclusion peut être étendue à tous les génocides. Dans des cas rarissimes, on parvient à condamner quelques-uns des chefs. La plupart du temps, les procédures s'éternisent (souvenons-nous du procès interminable de Slobodan Milosevic, mort de maladie avant qu'un verdict ait été rendu). L'immense majorité de ceux qui ont participé de façon coupable à un génocide jouissent quant à eux d'une impunité complète. Par exemple, des études anthropologiques dans l'ancienne Yougoslavie ont montré que les villageois se désignent les uns les autres avec raison comme des meurtriers et continuent d'habiter sur des côtés opposés de la même rue. On n'y peut rien faire. Il n'y a donc pas de quoi pavoiser sur les aptitudes de la justice pénale à régler les problèmes criminels les plus graves.

4. La recherche criminologique future va-t-elle nous permettre de sortir de ces impasses? Il ne faut certes pas désespérer ni méconnaitre les ressources de la criminologie. Toutefois, je m'interroge sur le futur de la criminologie d'expression française. Celle-ci est vivante et productive au Canada, où nous disposons d'institutions où se pratique cette discipline que nous n'hésitons pas à appeler criminologie. Toutefois, un nombre croissant de chercheurs francophones du Québec et du Canada choisissent de publier leurs recherches en anglais. On ne saurait les blâmer. C'est sans doute la seule façon pour eux d'assurer la diffusion et la discussion des résultats de leurs recherches et de leurs réflexions. En effet, pour des raisons que nous n'avons pas le temps d'élucider, la criminologie ne jouit pas d'une existence institutionnelle robuste en France et le débat criminologique y tombe généralement à plat. Les recherches les plus fécondes se poursuivent au sein d'un ensemble bien 
pesé d'appellations licites - l'histoire, la sociologie du crime et de la déviance, la sociologie du droit, la sociologie de l'État, les sciences politiques, la philosophie et la théorie du droit, l'économie et même l'anthropologie. Seule la criminologie semble exclue des agapes de l'université et fait même l'objet d'un ostracisme de bon goût. Toutefois, lorsque nos collègues français publient dans des revues en langue anglaise, ils ne répugnent pas à inscrire leurs travaux sous l'appellation de «criminology». Ils paraissent toutefois avoir méconnu pour eux-mêmes les effets rassembleurs d'une désignation disciplinaire conséquente. Il s'ensuit un émiettement dirimant de ce champ de recherche qui comprend le crime, la déviance et les pratiques de contrôle, alors que celuici constitue un espace théorique relativement intégré et fertile dans la plupart des autres pays. La criminologie et les savoirs afférents, comme les études policières, font donc l'objet d'un développement soutenu dans les pays anglophones et dans les pays européens non francophones. En France, on tombe d'IHESI en INHES. À moins d'un redressement que j'estime improbable, la criminologie d'expression française se fractionnera jusqu'à l'ensablement. Elle constituera à la limite une banlieue précieuse du savoir où des notables retirés dans leurs pavillons s'accuseront réciproquement d'être des parvenus jusqu'à ce qu'ils soient suffisamment lassés pour refermer leurs volets. 